1

2

13 Corresponding author: Angelo De Santis (angelo.desantis@ingv.it) magnetic Swarm satellites

${ }^{1}$ Istituto Nazionale di Geofisica e Vulcanologia, Roma, Italy.

${ }^{2}$ G. D’Annunzio University, Chieti, Italy.

${ }^{3}$ IAASARS, National Observatory of Athens, Athens, Greece.

${ }^{4}$ Centre National d'Etudes Spatiales, Paris, France.

* Now at Universidad Complutense de Madrid, Madrid, Spain.

2

\title{
Potential earthquake precursory pattern from space: the 2015 Nepal event as seen by
}

A. De Santis ${ }^{1,2}$, G. Balasis ${ }^{3}$, F.J. Pavón-Carrasco ${ }^{1 *}$, G. Cianchini ${ }^{1}$, and M. Mandea ${ }^{4}$

14 Key Points:

15 Earthquake; magnetic satellites; Swarm; Lithosphere-Atmosphere-Ionosphere Coupling 16 
17 Abstract

18 A large earthquake of 7.8 magnitude occurred on 25 April 2015, 06:26 UTC, with the epicenter

19 in Nepal. Here, taking advantage of measurements provided by the Swarm magnetic satellites,

20 we investigate the possibility to detect some series of pre-earthquake magnetic anomalous

21 signals, likely due to a lithosphere-atmosphere-ionosphere coupling, that can be a potential

22 earthquake precursory pattern. Different techniques have been applied to Swarm data available

23 during two months around earthquake occurrence. From the detected magnetic anomalies series

24 (during night and magnetically quiet times or with an automatic detection algorithm), we show

25 that the cumulative number of anomalies follows the same typical power-law behavior of a

26 critical system approaching its critical time, and hence recovers as the typical recovery phase

27 after a large event. The similarity of this behavior with the one obtained from seismic data analysis and the application of the analyses also to another period without significant seismicity do support a lithospheric-linked origin of the observed magnetic anomalies. We suggest that they might be connected to the preparation phase of the Nepal earthquake.

\section{Introduction}

The lithosphere, under the stress due to the plate tectonics, releases most of its energy with some rapid ruptures: the earthquakes (EQs). Intense shallow EQs are usually very destructive. Their

35 seismic release requires a long time of preparation before it occurs so suddenly, when a 36 particular fault cannot longer sustain the increasing deformation [e.g. Scholz 2002]. Recently, it 37 has been shown that the ionosphere keeps the signature of seismic fault shortly after an 
earthquake [e.g. Occhipinti et al., 2013, and references therein]. This offers the possibility to retrieve seismic information from ionospheric observations.

An important and debated question arises about the possibility that, during the phase of EQ preparation, electromagnetic waves and/or particles could be transferred from the solid Earth (in particular the lithosphere) to the atmosphere, with a particular effect in the ionosphere, above around 50 km [e.g. Pulinets and Boyarchuk, 2004; Freund, 2011; Pulinets and Ouzounov, 2011; De Santis et al., 2015]. One of the most general models of coupling is based on the emission of a radioactive gas [Pulinets and Boyarchuk, 2004] or metallic ions [Freund, 2011] before a large earthquake, which may change the distribution of electric potential above the surface of the Earth and then up to the ionosphere [e.g., Pulinets and Boyarchuk, 2004; Sorokin et al., 2001]. Penetration of the electric field to the ionosphere could produce ionospheric plasma density and/or conductivity anomalies, which are observed above seismic zones [e.g., Liu et al., 2006; Kon et al., 2011]. An alternative explanation is that the radon emitted before an earthquake would increase the conductivity of air at ground level and that the ensuing increase of current in the fair weather global circuit would lower the ionosphere [Harrison et al. 2010]. Therefore, it is expected that low Earth orbiting (LEO) satellites could be the best possible dedicated platforms of sensors to detect any electromagnetic, acoustic or infrared seismic-linked precursors. Certainly, space observations have to be investigated together with ground (and near-surface) seismic and other geophysical observations, in order to have a more complete picture of the possible involved phenomena. 
60 Few attempts have been made to deeply investigate pre-EQ ionospheric perturbations. The

61 French satellite mission, DEMETER (Detection of Electro-Magnetic Emissions Transmitted

62 from Earthquake Regions), was suitably designed and launched (2004-2010) for searching

63 electromagnetic anomalies likely anticipating large earthquakes [Parrot, 1995]. Systematic

64 analyses over parts of the mission data [Němec et al., 2008] or the full life-time of satellite [Piša

65 et al., 2013; Li and Parrot, 2013] have showed a significant statistical correlation between

66 ionospheric anomalies and large events, within a few days before the event, although no

67 undisputed precursor evidence has been proved.

68

69

70

71

72

73

74

75

76

77

78

79

80

81

82

The opportunity to investigate the ionosphere perturbation is currently due to the new Swarm constellation [Friis-Christensen et al., 2006]. From the end of 2013 the three Swarm satellites measure the Earth's magnetic field with an unprecedented accuracy. Since then, a few large EQs occurred, but here we focus on the EQ occurred on 25 April 2015, 06:26 UTC, in Nepal region, $77 \mathrm{~km} \mathrm{NW}$ of Kathmandu, with a magnitude Mw 7.8 (hypocenter $28.15^{\circ} \mathrm{N}, 84.71^{\circ} \mathrm{E}$ at a depth of $15 \mathrm{~km}$ ), in an area characterized by a persistent seismicity [Parameswaran et al. 2015]. According to the USGS, the fault rupture plane was $295^{\circ}$ and the dip $10^{\circ} \mathrm{NNE}$; the rupture surface was approximately $100 \mathrm{~km}$ along the strike and $80 \mathrm{~km}$ along the downdip $(135 \mathrm{~km} \times 100$ km, according to $W u$ et al., [2016]). Seventeen days later (May 12, 2015), a strong aftershock followed, approximately $77 \mathrm{~km}$ NE of Kathmandu, with a smaller estimated magnitude of Mw 7.3 [Parameswaran et al. 2015].

1 This very damaging EQ has been studied using different geophysical datasets. For example,

82 Galetzka et al. [2015] performed a detailed geodetic imaging of earthquake ruptures. They used 
Global Positioning System (GPS) and interferometric synthetic aperture radar (SAR) data to model the earthquake rupture and found that the smooth slip onset caused moderate ground shaking at high frequencies $(>1 \mathrm{~Hz})$ and minimized damage to vernacular dwellings, while whole-basin resonance at a period of 4 to 5 seconds caused the collapse of tall structures. Chen et al. [2016] found a significant gravity anomaly before the EQ which could be an important precursor; indeed, the broad source region derived from gravity data is consistent with the idea that a broad region of crust in southern Tibet is involved in the storing of elastic strain energy driving the Himalayan earthquakes [Feldl and Bilham, 2006]. Another intriguing feature comes from some atmospheric parameters, such as the aerosol optical depth (AOD) and the columnar ozone (NO2) found to be higher than $40 \%$ and $6 \%$, respectively prior to the occurrence of the earthquake [Galguly, 2016]. The UV aerosol index, AOD and the columnar NO2 increased, while columnar ozone and sea level pressure dropped following the earthquake. This result, if confirmed, might support a coupling between the lithosphere and the atmosphere, during the phase of preparation of the Nepal EQ. Finally, let us note that this EQ seems to have released only a fraction of the energy still trapped in the underlying fault, so that the area has still the potential to host another large earthquake in the near future [Avouac et al., 2015].

In this paper, we explore a possible pre-EQ lithosphere-atmosphere-ionosphere (LAI) coupling by analyzing magnetic and ionospheric data from the Swarm satellites, applying different methods over different intervals of time around the 2015 Nepal EQ occurrence. Firstly, we shortly describe the used Swarm data, together with ground seismic data, and the applied methods. In the following, we present the obtained results based on: i) the comparison of the average power in Pc3 frequency band (0.022-0.1 Hz; Saito, [1969]) for a 2 month period in 2015 
106 with that of the previous year, when no large earthquake occurred in the area of interest; and ii)

107 the search for the single "anomalies" and then of the temporal behavior of the cumulative

108 number of anomalies. Finally, we specify the main results based on a comparison from the latter

109 analysis with an analogous method applied to the ground seismic data, and a confutation analysis

110 to the Swarm magnetic data in another no significant seismic period, before concluding.

\section{2. Data}

113

114

115

116

117

118

119

121

122

123

124

125

126

127

\section{Swarm satellite observations}

Swarm is an ESA (European Space Agency) satellite mission of three satellites devoted to the study of the geomagnetic field and its dynamics [Friis-Christensen et al., 2006]. The main objectives of the mission are to deliver the best survey of the geomagnetic field and its temporal variation, and to acquire a characterization of the internal field sources and of the ionosphericmagnetospheric current systems, due to the very high accuracy of the onboard instruments ${ }^{1}$. This constellation was designed to derive the first global representation of the geomagnetic field variations on times scales from an hour to several years, addressing the crucial problem of source separation.

\section{The three Swarm satellites are identical, and the constellation consists of two satellites (A and C)} flying almost side-by-side (longitude separation of $1.4^{\circ}$ at equator) at an altitude close to $450 \mathrm{~km}$, and inclination of $87.4^{\circ}$. The third satellite (B) flies above, close to $510 \mathrm{~km}$, on a more polar orbit (inclination of $88^{\circ}$ ) and has allowed for a progressive Local Time (LT) separation with respect to A and C, of about three hours, in the beginning of 2016. The three satellites are also named

\footnotetext{
${ }^{1}$ http://esamultimedia.esa.int/docs/SP_1279_6_Swarm.pdf
} 
Alpha, Bravo and Charlie. This specific constellation allows the scientists to observe the small space-scale variations of the geomagnetic field, particularly those linked to the lithospheric field. The main mission sensors are a couple of magnetometers, i.e. an Absolute Scalar Magnetometer (ASM) providing, in nominal mode, measurements of the field intensity and a Vector Field Magnetometer (VFM) mounted halfway along the boom on an optical bench together with the Star Trackers, providing field directions. Other sensors complete the payload of each satellite, in particular, two electric field and particle sensors (Langmuir Probes), a GPS antenna and an accelerometer.

The previous magnetic satellite missions were single satellite systems. With Swarm data taken at three different orbits and two altitudes, it is possible to investigate for distinctive seismogenic signatures in the ionosphere from non-seismic external (ionospheric and/or magnetospheric) electromagnetic signals, providing that proper analysis are applied in order to extract the wealth of information that underlies the data.

In this study we have analyzed the magnetic VFM (Low Resolution) and the ASM Level1B 1Hz data $^{2}$, and the electron density $\mathrm{Ne} 2 \mathrm{~Hz}$ data $^{1}$, along the tracks that crossed a circular region within $\mathrm{R}=2750 \mathrm{~km}$ from the Nepal EQ epicenter. The distance $\mathrm{R}$ is the so-called Dobrovolsky radius strain [Dobrovolsky et al., 1979], $\mathrm{R}=10^{0.43 \mathrm{M}}$. This distance was initially defined as the distance from the impending EQ fault where the strain due to the preparation EQ phase reaches the terrestrial tides background level. Some later works (e.g. Plastino et al., 2002; Vizzini and Brai, 2012) showed that precursory phenomena occur within this distance. Since, an uncertainty of around 0.2 units is noted in the estimated magnitude as given by different agencies (e.g. while

\footnotetext{
${ }^{2} \mathrm{ftp} / / /$ swarm-diss.eo.esa.int
} 
151 USGS estimated M7.8, the Chinese Earthquake Administration provided M8.1; Zao, 2016),

152 here, we have considered a Dobrovolsky area corresponding to a magnitude $\mathrm{M}=8$.

153

154

155

156

157

158

159

160

161

162

163

164

165

166

167

168

169

170

171

172

\section{Seismic Data}

Seismic catalogues represent fundamental data sources for earthquake and seismic hazard studies: they contain the earthquake source parameters (e.g. origin time, hypocenter, magnitude etc.) and may be created by single Countries or international Agencies. For this study, we have used data extracted from the USGS Catalog ${ }^{3}$ : in particular, we have collected events from 1 April to 10 May, 2015 with magnitude M4+ occurred within the above defined Dobrovolsky region.

\section{Results}

Considering the available Swarm data around the time of EQ, two specific analyses have been performed, and they are presented here.

\subsection{Pc3 activity in 2014 and 2015}

Magnetospheric ultra low frequency (ULF) waves in the topside ionosphere are typically transmitted from magnetospheric and upstream solar wind sources. Oscillations with quasisinusoidal waveform are called pulsations continuous (Pc). Those with waveforms that are more irregular are named pulsations irregular $(\mathrm{Pi})$ and are associated with magnetospheric substorms. In particular (Jacobs et al., 1964), continuous pulsations with frequencies in the range $1 \mathrm{mHz}$ to 5 Hz, denoted as Pc1-2 (100 mHz-5 Hz), Pc3 (20-100 mHz), Pc4 (7-20 mHz), and Pc5 (1-7 $\mathrm{mHz}$ ), have been extensively studied using measurements from both space-borne and ground-

\footnotetext{
${ }^{3}$ http://earthquake.usgs.gov
} 
173 based instruments for many years (for a recent review see Menk, 2011). They are broadly of two

174 types, depending on whether their energy source originates in the solar wind on the dayside or

175 from processes within the magnetosphere (e.g., substorms and other instabilities in the 176 magnetotail) on the nightside.

177

178

179

180

181

182

183

184

185

186

187

188

189

190

191

192

193

194

195

The low Earth orbit (LEO) CHAMP satellite has been one of the most successful missions for the study of the Earth's magnetic field, with high-sensitivity and accuracy magnetometer measurements orbiting within an altitude range 450-300 km for more than a decade (July 2000September 2010). For the first time long-term statistical studies on the occurrence of ULF wave events in the topside ionosphere were possible. LEO observations of ULF waves can only be reliably done and without too much spatial aliasing for the Pc1/Pi1 and Pc2/3 waves (frequency $\sim 22-100 \mathrm{mHz}$ ). Due to the fast motion through field lines in a LEO orbit lower frequency Pc4-5 waves $(1-10 \mathrm{mHz})$ cannot be accurately determined by LEO satellites, their period being longer than the spacecraft transition time through the wave region (Heilig et al., 2007; Balasis et al., 2012). Though Pi2 waves have lower frequencies (frequency 2-25 mHz), thanks to their large spatial scales at low latitudes, they have also been detected by LEO satellites. A spatial scale of Pi2 waves along an orbit becomes comparable with the scale of lithospheric magnetic anomalies (Maus et al., 2007). Therefore, to reveal reliably Pi2 signatures from LEO satellite data the knowledge of a detailed magnetic model of the Earth (e.g., Maus et al., 2007) is necessary. Therefore, the most prominent LEO observations of ULF waves are usually made in the Pc3 frequency range $(20-100 \mathrm{mHz})$ as also show recent studies using data from the Swarm mission [Balasis et al., 2015; Heilig and Sutcliffe, 2016]. 
196 There have been several studies suggesting that ULF pulsations may be associated with

197 earthquakes [Fraser-Smith et al., 1990; Hayakawa et al., 1996]. The majority of these studies

198 refers to the detection of these signals in ground-based magnetometer measurements [Hayakawa

199 et al., 2015; Contoyiannis et al., 2016; Potirakis et al., 2016 and references therein]. On the other

200 hand, there is only a handful of studies that have been attempted to correlate ULF pulsations with

201 seismic activity from space-borne measurements [e.g., Balasis and Mandea, 2007 for CHAMP

202 satellite and Walker et al., 2013 for DEMETER satellite].

In this analysis, we have used the low resolution ASM data with a sampling rate of $1 \mathrm{~Hz}$ and of $50 \mathrm{sec})$ to $100 \mathrm{mHz}(10 \mathrm{sec})$. An electromagnetic wave of higher frequency, e.g. at $200 \mathrm{mHz}$ data points, making the analysis statistically questioning. of daily power spectra, while the second turns to the actual wave detection. For every day in the

211 time interval of interest, i.e., approximately one month before and after the Nepal EQ both in 2122014 and 2015: this time interval could have been extended into a longer time period, however, 213 the neighboring months of March and June 2015 were both dominated by strong geomagnetic 214 activity associated with two intense magnetic storms occurred one at each month as witnessed by 215 the Dst index ${ }^{4}$.

216 The hourly Disturbance storm-time (Dst) geomagnetic activity index is computed from an 217 average over 4 mid-latitude magnetic observatories, and hence serves as a proxy for the

\footnotetext{
${ }^{4}$ http://wdc.kugi.kyoto-u.ac.jp/dst_provisional/201503/index.html; http://wdc.kugi.kyoto-u.ac.jp/dst_realtime/201506/index.html
} 
magnetospheric ring current, and thus magnetic storm occurrence [http://wdc.kugi.kyotou.ac.jp/dst_realtime/presentmonth/index. html]. The two intense magnetic storms occurred on 17 March and 23 June 2015 with minimum Dst index of -223 nT and -204 nT, respectively. Then, we have performed the following analysis. First, the magnetic data are downloaded, along with the files of the previous and the next day. Since all spectral methods are plagued by edge effects [Torrence and Compo, 1998], we have appended the magnetic field time series of the current day, with a few hours from the previous and next day. Whatever edge effects appear they might affect these additional "margins", which can be then safely removed from the process, getting the spectral data of the day under processing clean of edge effects. From the magnetic time series of the ASM instrument we have derived the magnitude of the magnetic field and applied a Chebysev type II, zero-phase, high-pass filter with a cutoff frequency of $20 \mathrm{mHz}$ to remove all lower frequency components from the signal and bring the Pc3 range in the spotlight [Williams and Taylors, 1988]. Using the wavelet method, with the Morlet mother function, we have produced the power spectrum of the magnetic field series, for 50 logarithmically spaced frequencies from 20 to $100 \mathrm{mHz}$ [Balasis et al., 2013] and removed the aforementioned margins. In parallel to that, the positional vector of the spacecraft is extracted from the files and converted to magnetic coordinates (magnetic latitude, longitude and local time), as well as the electron density series from the Swarm electric field instrument (EFI) data files of the same day. The final spectrum, along with the time series of the magnetic field, the electron density and all positional information are then exported in a daily file and saved in the database.

The second step refers to the wave detection, which begins by reading the daily output files and segmenting them in tracks (half-orbits) from -90 to +90 degrees in magnetic latitude. For each 
241 such track, the maximum power per second (stored in the spectrum) is calculated and all

242 segments of consecutive points that exceed a threshold of $0.5\left(\mathrm{nT}^{2} / \mathrm{Hz}\right.$ ) (which roughly

243 corresponds to a pulsation with a minimum amplitude of $0.15 \mathrm{nT}$ ) are labeled as "candidate

244 events". Each candidate is tested against a series of criteria that help rule out artificial signals that

245 might result from instrument or telemetry errors. As such, for the candidate event to not be

246 discarded it must i) exhibit a duration at least two times its peak period, ii) have an amplitude

247 that does not exceed a given limit (10 nT) and iii) be smooth enough to constitute a continuous

248 pulsation, so its difference series must always be smaller than $1 \mathrm{nT}$. In order to avoid traces of

249 activity from lower Pc classes (below $20 \mathrm{mHz}$ ) that have not been completely eliminated by the

250 filtering process, the peak of the wave activity has to be at a frequency that does not lie at the

251 limits of the examined range, so only pulsations with a peak frequency that lies well above 21

$252 \mathrm{mHz}$ are accepted. Lastly, if the candidate is detected when the satellite is located at the nightside

253 sector, an additional criterion must be met, namely that its electron density series must not

254 display abrupt changes. If it does, then the candidate is classified as belonging to the Plasma

255 Instability class, which includes (but is not limited to) equatorial spread-F (ESF) events [Stolle et

256 al., 2006] at low latitudes and activity related to field aligned or auroral currents at higher

257 latitudes. If the electron density profile is smooth enough, or the wave event takes place at the

258 dayside sector then it is classified as a Pc3 Wave.

260 For the production of wavemaps the coordinate space, i.e., geographical latitude vs longitude is 261 divided into a 50 by 50 grid, which provides a resolution of 3.6 degrees in latitude and 7.2 262 degrees in longitude. For every second in the duration of an event, its total Pc3 power is 263 calculated and added to the appropriate grid point that the measurement belongs, based on the 
264 satellite location at the same moment, thus allowing the entire extent of the wave to be accurately

265 represented. In order to make the resulting maps less dependent on the satellite orbit (the number

266 of times it passes from each sector of local time) we have divided the summed power by the

267 number of seconds the satellite spends in each grid point.

269 Having produced the wavemaps for the months April to May 2014 and 2015, we then have 270 divided them, point by point, to remove the wave power due to a normal Pc3 wave activity, 271 which should be statistically similar in both cases reflecting a period of quiet geomagnetic 272 activity as in our case. Figure 1 shows, on global scale, the difference in the Pc3 wave power 273 between 2015 and 2014, as estimated from Swarm A and Swarm B data, limited to 1 April to 31 274 May of each year. In the map based on the Swarm A data we notice an anomalous difference just 275 above the Nepal EQ epicenter and its magnetic conjugate point. On the map based on Swarm B 276 no clear anomaly is noticeable in this area, probably because this latter satellite is at a higher 277 altitude, and, supposing a simple model of lithospheric source with geometrical attenuation with 278 the distance, the effect is dramatically minimized. 

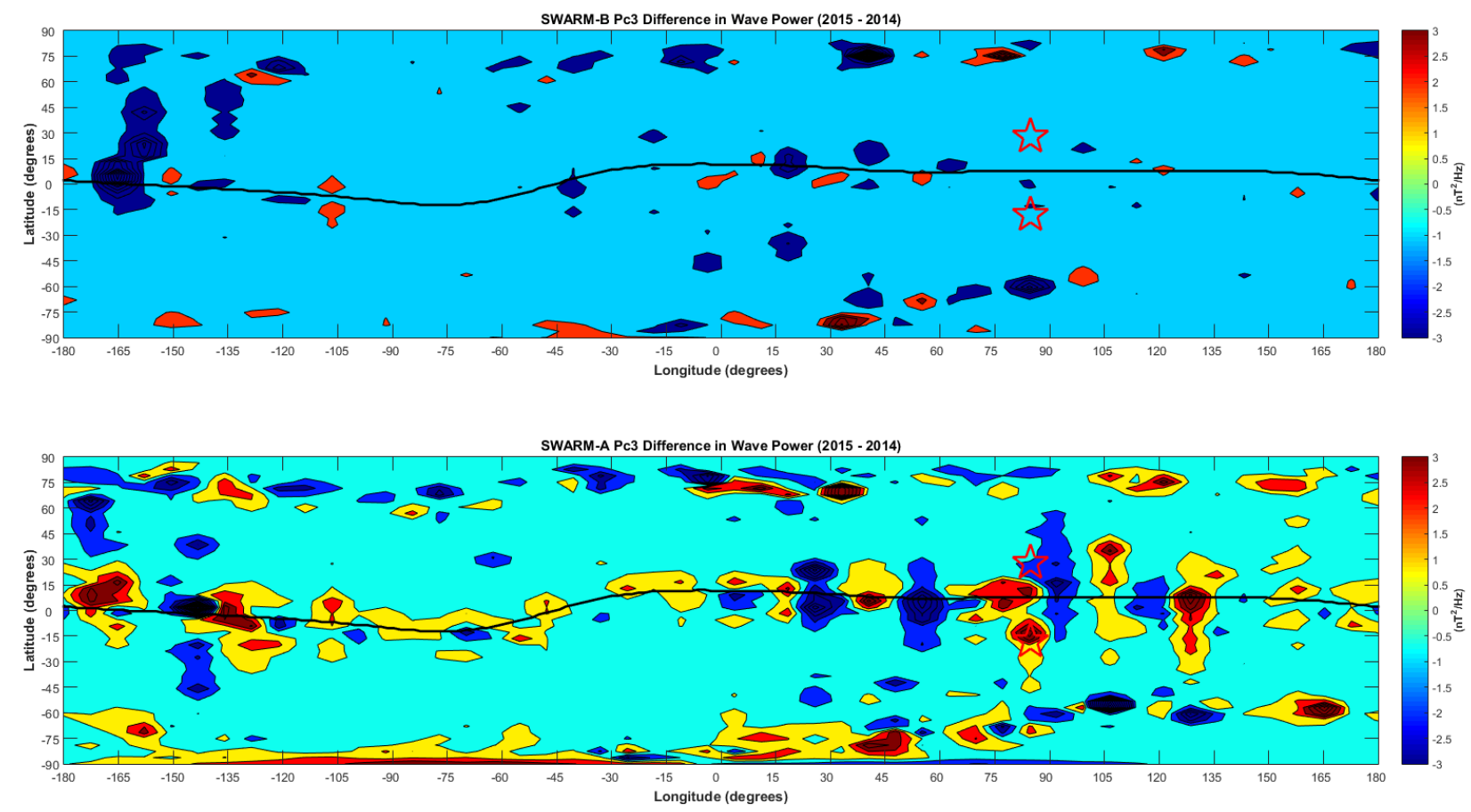

280 Figure 1. Pc3 difference in Wave Power 2015-2014 for Swarm A satellite (bottom) and Swarm B satellite (top), both 281 cases, in the period 1 April to 31 May of each year to avoid periods of magnetic storms. The northern hemisphere 282 and southern hemisphere red stars are the positions of the 2015 Nepal EQ epicentre and its conjugate point,

283 respectively. The black line indicates the magnetic equator at both panels.

285 Please note that, by construction, the wavemaps depict average values of the wave activity for 286 the entire two-month period that was examined. Thus, the activity from many local times that 287 correspond to the same latitude-longitude grid point will have been averaged together and so it is impossible to extract the notion of local time from these graphs. An alternative approach would

289 be to construct the maps on a latitude vs local-time grid, which would show any such behavior, 290 but in this case activity from many longitudes would have been averaged together, thus making it 291 impossible to associate any observations with the EQ epicenter. We think the way we chose to 292 construct the above wavemaps is the best compromise. 


\subsection{Analysis of magnetic satellite data and seismic data}

Previous analysis points toward a possible coupling between lithosphere and ionosphere. To verify this assessment, we have performed another study to enforce the idea of a coupling between lithosphere and ionosphere occurring before the large Nepal EQ.

300 We have performed an analysis on the vector data provided by Swarm A. We have considered all 301 magnetic X (North), Y (East), Z (vertical) and F (field intensity) measurements and computed 302 the first time difference along the night orbital tracks (between 2200 to 0600 LT). This procedure 303 removes most of the slow fluctuations of the magnetic signal. Then, we have adjusted and 304 removed the best fit curve obtained by a series of cubic-splines with knot points every $20 \mathrm{~s}$ and plot the obtained track residuals. These residuals are very sensitive to any abrupt change in the original signal, or in its slope. Finally, we have examined the obtained plots to identify clear 307 anomalies with the following characteristics: i) peak-to-peak amplitude $\geq 0.3 \mathrm{nT} / \mathrm{s}$ and 308 persistence for at least ten seconds (so well inside the ULF band); ii) occurrence during very 309 magnetic quiet night-times (the absolute value of the Dst index lower than $10 \mathrm{nT}$ and $\mathrm{a}_{\mathrm{p}} \leq 12$ $310 \mathrm{nT})^{5}$; iii) situated within the Dobrovolsky circular area around the epicenter. We notice that the 311 best clear anomalies generally occur in the Y-component. Once identified, we plot for each 312 anomaly, at its occurrence time $t$, the subsequent cumulative number, $N(t)$, i.e. the order of the $N$ 313 th earthquake until time $t$. An example of chosen track with an anomaly is shown in Figure 2a, 314 while Figure 2b shows as example a track without significant anomalies.

\footnotetext{
${ }^{5}$ https://www.ngdc.noaa.gov/stp/geomag/indices.html
} 
Sat. Alpha. Y/M/D:2015/4/14. Track \# 2. meanLT : 5:07:47. meanUTC : 0:31:41. Dst=2. ap=2

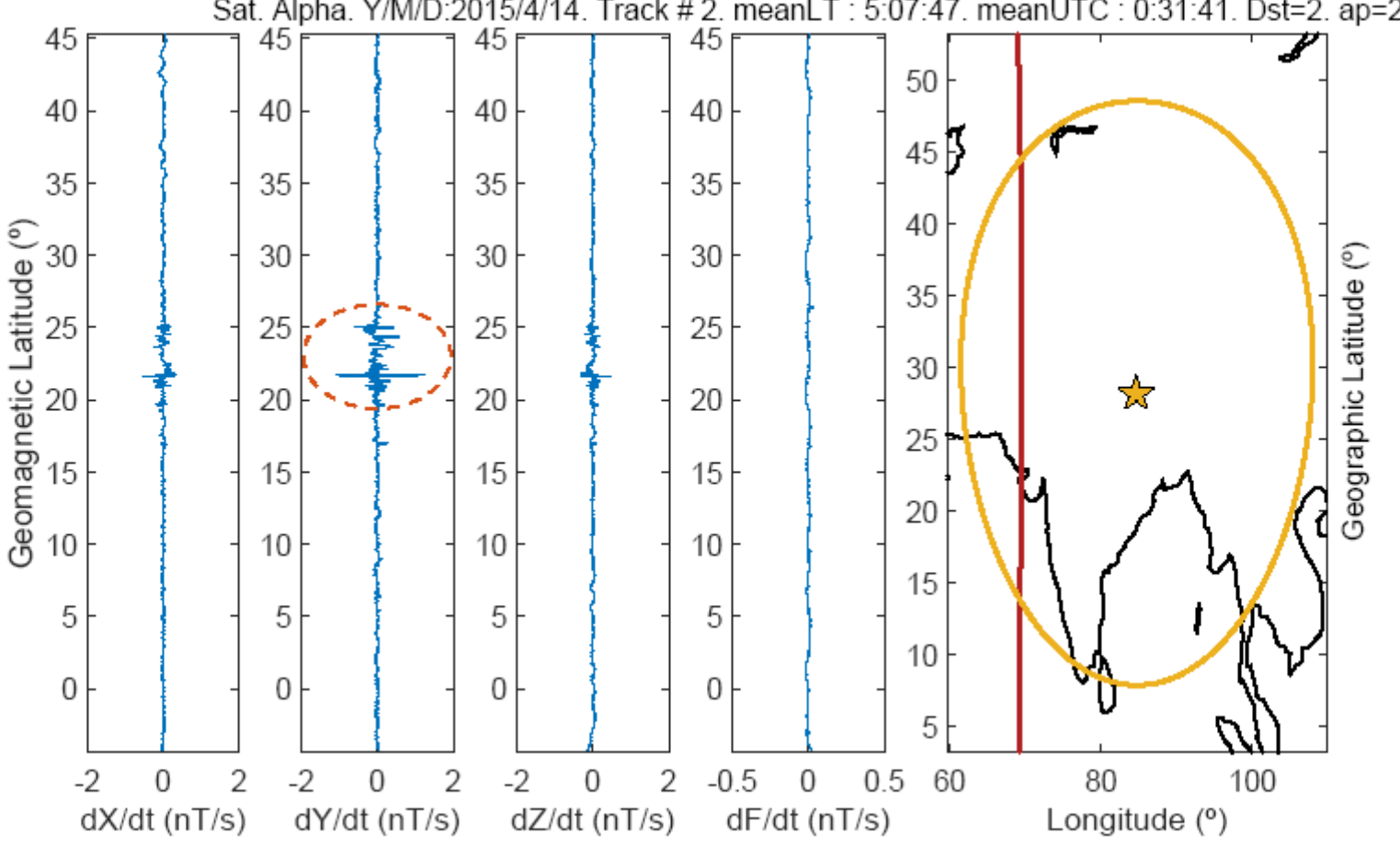

Figure 2a Specific plots $(\mathrm{dX} / \mathrm{dt}, \mathrm{dY} / \mathrm{dt}, \mathrm{dZ} / \mathrm{dt}$ from fluxgate magnetometer and $\mathrm{dF} / \mathrm{dt}$ from scalar magnetometer of Alpha satellite; and geographic map of the satellite track where the yellow star is the EQ epicentre) of one of the corresponding chosen night-time tracks with anomalies used in Fig 3 (bottom). The presumed anomaly is evidenced by a red oval. Above are indicated: date, \# of track (half-orbit), mean Local time, UTC, and the values of the geomagnetic indices Dst and $a_{p}$. 


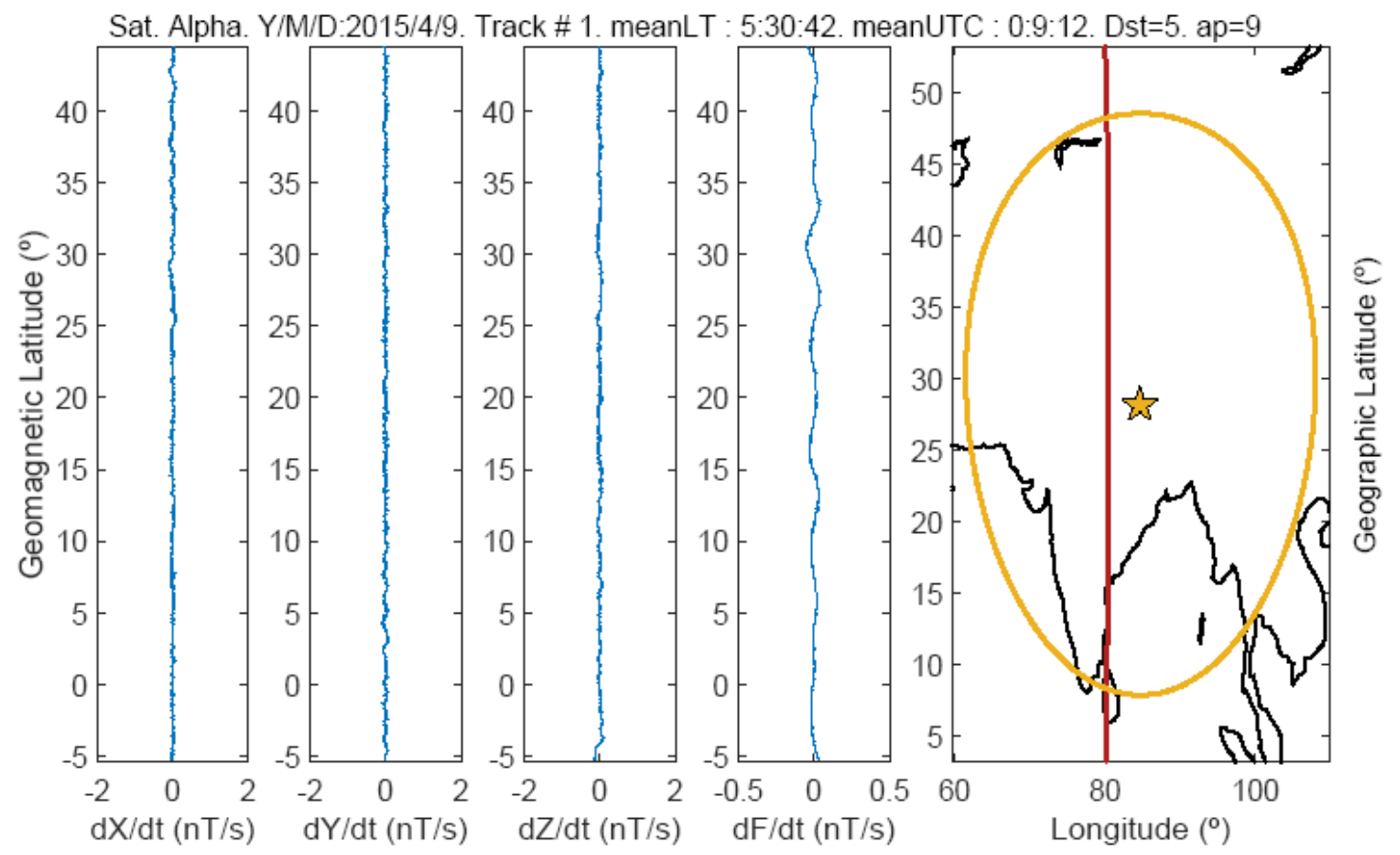

Figure $2 b$ Specific plots $(\mathrm{dX} / \mathrm{dt}, \mathrm{dY} / \mathrm{dt}, \mathrm{dZ} / \mathrm{dt}$ from fluxgate magnetometer and $\mathrm{dF} / \mathrm{dt}$ from scalar magnetometer of Alpha satellite; and geographic map of the satellite track where the yellow star is the EQ epicentre) of a night-time track without anomalies. Above are indicated: date, \# of track (half-orbit), mean Local time, UTC, and the values of the geomagnetic indices Dst and $\mathrm{a}_{\mathrm{p}}$.

This technique is used because of its sensitivity to anomalous deviations with respect to a typical random process whose cumulated value has a statistical linear increase. In particular, in case of critical phenomena we would expect more frequent anomalies when they approach the critical point, and less frequent anomalies after, so providing an accelerating power law before it and a decelerating recovery after, such as:

$$
N(t)=A+B\left|t_{f}-t\right|^{m}
$$

with $A$ and $B$ two constants, and $0<m<1$ or $m>1$ in the acceleration or deceleration phase, respectively [e.g. De Santis, 2014]. 
339 The bottom of Figure 3 shows the temporal behavior of $N(t)$, denoted here as $N$ (magnetic

340 anomalies). We indeed notice a power-law temporal behavior before the EQ (its occurrence is

341 indicated by a vertical dashed line) with an upper concavity and a subsequent power-law

342 behavior after, with an opposite concavity. Same figure shows the best fit power-laws before or

343 after the Nepal main-shock. This behavior is compared with the analogous of the cumulative

344 number of M4+ earthquakes, $N(\mathrm{EQs})$, occurred before and after the Nepal EQ, in the

345 Dobrovolsky region. The two plots are very similar, both identifying the phases before and after

346 the large EQ, and supporting the hypothesis that the noticed magnetic anomalies in Swarm data

347 are mostly of internal origin, due to a LAI coupling. This process can be linked to the preparation

348 phase of a large EQ and on the aftershocks phase [Pulinets and Boyarchuk, 2004; Pulinets and

349 Ouzounov, 2011].
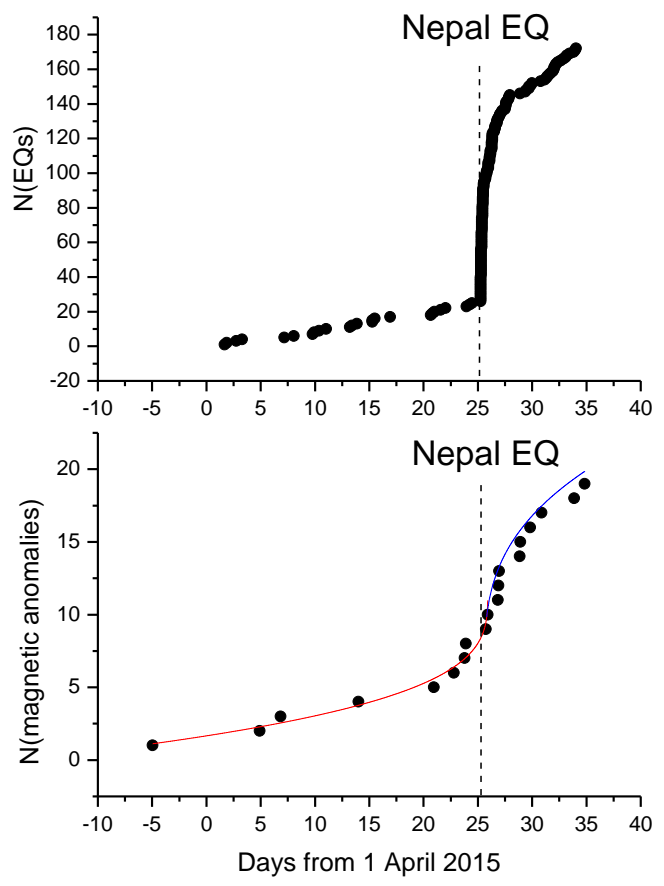

351 Figure 3 Cumulative number of magnetic anomalies detected by Swarm satellite A versus time. Red and blue curves 352 are the best power-law fits before and after the Nepal main-shock, respectively (bottom); Cumulative number of 353 M4+ earthquakes occurred within Dobrovolsky area versus time (top). 
355 The cumulative count of the magnetically quiet tracks (Figure 4) indicates a similar behavior, 356 especially after the EQ. This means that our previous result could be due, in some unknown 357 amount, to a modulation of the daily distribution of quiet times intervals used in the satellite data 358 selection.

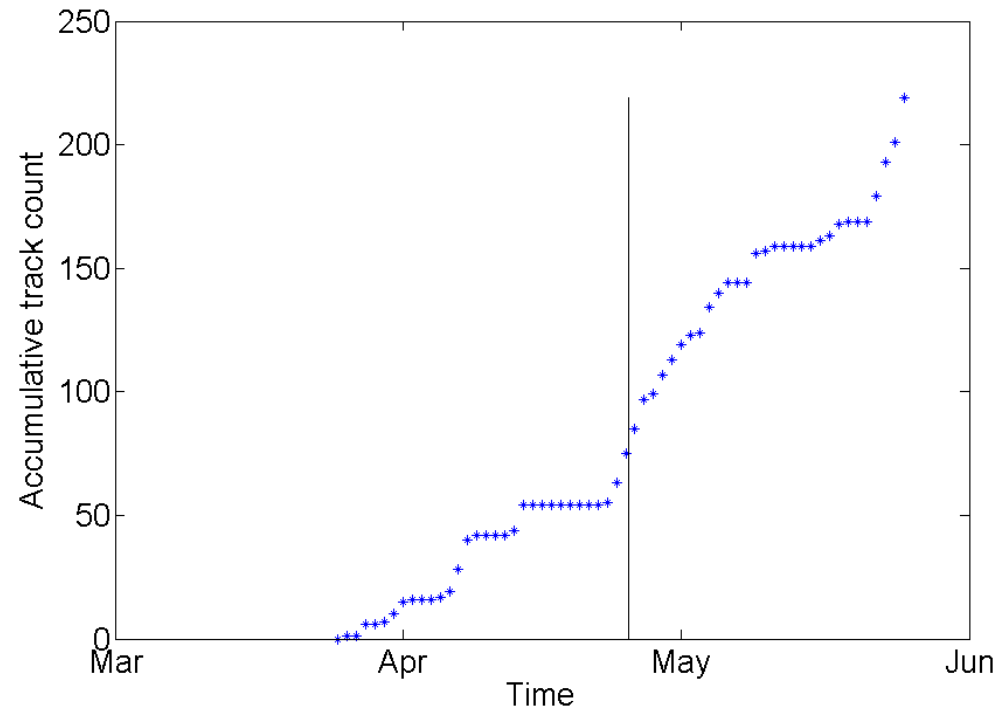

Figure 4. Cumulative number of magnetically quiet satellite tracks.

This possibility cannot be fully excluded so a more robust approach is required. The proposed one is based on an automatic selection of anomalies, only on the basis of a given number of standard deviations, with no consideration of night or day, quiet or disturbed times. As partial justification of relaxing the quite time criterion of selection, we remind the recent paper by Currie and Waters [2014], which refutes the widely spread misbelief that ULF variations and geomagnetic activity are closely correlated, rather it leaves space to the assumption that sometimes ULF activity may be related to internal lithospheric processes, like, for instance, 
369 microcracks, shallow fluid migration and/or particle emissions associated with earthquake 370 preparation phase.

371 When considering, independently from the quiet index selection, only those anomalies above 3.5 372 standard deviations (with a standard deviation estimated along the entire track from $55^{\circ} \mathrm{N}$ to $55^{\circ} \mathrm{S}$ 373 geomagnetic latitude, to avoid the auroral and polar disturbances), we obtain almost the same 374 type of "critical" curve (Figure 5), at the cost of less detected anomalies, because of the more 375 stringent criterion of selection. Let us note that: i) when two detected anomalies occur in the 376 same day, we indicate it as one single point, but we add two counts in the cumulative value (this 377 explains why, although the detected anomalies are 17, there are 11 points in the plot); ii) 378 although most of anomalies (15 out of 17) occur during rather quiet magnetic times (both |Dst| 379 and $a_{p}$ less than $20 \mathrm{nT}$ ), there are two exceptions on the same day (i.e. 17 April 2015) with anomaly occurrence during some slightly perturbed periods (Dst=-34 and $-36 \mathrm{nT}$, although $\mathrm{a}_{\mathrm{p}}=7$

381 nT). This means that even in a perturbed day, the detected anomaly clearly emerges.

In Figure 5, for simplicity, instead of two power-law functions before and after the main-shock,

384 as (1), the complete "critical curve" around the large earthquake is approximated with a 385 generalized sigmoid function:

$$
S(t)=a+\frac{b-a}{\left(1-e^{\frac{t-t_{f}}{c}}\right)}
$$



function.

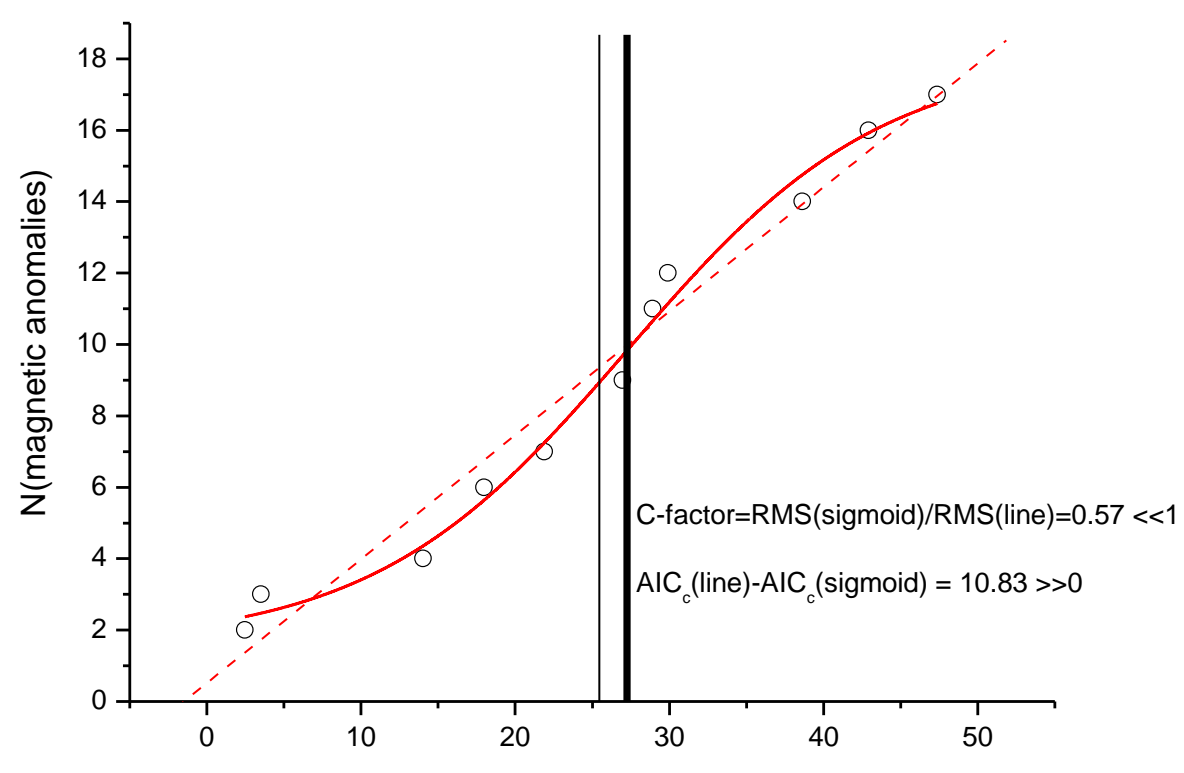

Day from 31 March 2015, 00:00 UT

391

392

Figure 5. Cumulative number of magnetic anomalies (empty circles) which are above 3.5 standard deviation. Red curve is the best sigmoid fit over the experimental data while the dashed line is the best linear fit. Vertical thin line is the time of the Nepal earthquake, while the vertical thick line is the predicted time given by the sigmoid fit. Both Cfactor and the corrected Akaike information point to a sigmoid function as a fitting model, better than the linear one.

Although this function is not a rigorous solution for a dynamical equation satisfying the approach of a system to its critical point, it is a sound representation of the correct solution, with the advantage of no singular point in its first time derivative. We have performed the fit in a unique solution over all periods of time around the main-shock, without the intention of making predictions of the impending EQ from the precursory magnetic anomalies, rather to invoke a LAI coupling. 
404 Another advantage of using the sigmoid fit is the estimation of the time $t_{f}$. Despite the used

405 function is only approximated, the parameter $t_{f}$ is a reasonable estimation of the time of the 406 radical change of the critical dynamical system, i.e. its critical point. In this specific case, the 407 obtained values are: $t_{f}=27.26( \pm 1.37)$ days from April $1^{\text {st }}, 2015$, that is surprisingly close to the 408 actual time (25.27 days) of the Nepal EQ. Certainly, this complete analysis can be performed 409 only once the event is occurred, because the sigmoid needs to be computed over a significant 410 time around the EQ. To understand whether the fit with the sigmoid function is robust, we have 411 compared it with that of a straight line. The sigmoid fit is clearly better than the linear one: their 412 Pearson's correlation coefficients, $r_{p}$, are 0.9950 and 0.9848 , respectively. In addition, we have 413 introduced the $C$-factor as the ratio between the RMS (root mean squares) of the sigmoid fit and 414 the RMS of the best line (e.g. Bowman et al., [1998] define the C-factor for a power-law and a 415 line) or equivalently:

$$
C=\sqrt{\left(1-r_{p}^{2}[\text { sigmoid }]\right) /\left(1-r_{p}^{2}[\text { line }]\right)}
$$

419 The lower than 1 the $C$-factor is, the better performance of the sigmoid fit with respect to the 420 linear one is obtained. In the present case, with $C=0.57$, it appears that the fit of the sigmoid 421 function is significantly better than the linear fit. Though the sigmoid function contains four parameters instead of the two for a line, we can prove that it is more efficient than the line, in terms of the corrected Akaike information $\mathrm{AIC}_{\mathrm{c}}[$ Akaike, 1973; Cavanaugh, 1997]: the corresponding criterion affirms that the best function is the one with lower $\mathrm{AIC}_{\mathrm{c}}$. Thus, we have calculated $\mathrm{AIC}_{\mathrm{c}}$ for both fitting functions, and represented in 
427 Figure 5. For the two functions we have found that $\mathrm{AIC}_{c}($ line $)>>\mathrm{AIC}_{\mathrm{c}}($ sigmoid $)$, confirming that 428 the sigmoid function is more appropriate to model the data than a simple linear fit. If the 429 cumulative number of magnetic anomalies is reproducing the effect of the LAI coupling before 430 and after a large EQ, the sigmoid fit also provides an estimate of the time of change of this 431 coupling, i.e. the time of occurrence of a large EQ.

\section{Confutation analysis}

The region of interest that includes the Dobrovolsky area around the 2015 Nepal EQ is very seismic and it is rather difficult to find long periods of complete seismic inactivity. Table S1 (Supplemental Material) shows, in the interval 1 Jan. 2014- 30 June 2016, the 3 periods with no M5.5+EQs, which are longer than 60 days. Here, we present the results of a confutation analysis for a non seismic situation: i) the wavelet power analysis of the Swarm magnetic data as observed all over the world over the same two months April and May, but in another year (2016) and ii) a track-by-track anomaly detection in the same region (Dobrovolsky area for the Nepal EQ), but in another 60-day period. In the former case no large M6+ seismicity was present in the considered Dobrovolsky area, while in the latter no M5.5+ earthquakes occurred (Table S1). Figures S1 and S2 (Supplemental Material) show the comparison of the wavelet power result in 2015 with that in 2016 and then the wavelet power result in 2014 with that in 2016, respectively. If we examine the area around the Nepal EQ epicentre and its conjugate point, we notice that the regional anomaly found in Alpha satellite, in the comparison 2015-2014 is still present in 20152016, while it is not present in the comparison 2014-2016, confirming the uniqueness of the anomaly in the period of time before the EQ. Regarding the second kind of analysis, and looking at the Table S1, excluding the second period, that includes the analysed EQ, and the third that is 
450 too close with the aftershocks activity of the same EQ, it remains only the first period useful for

451 the confutation analysis of this kind of method, i.e. from 1 June to 30 July 2014. We call this

452 dataset as "false" case, because no significant M5.5+ earthquakes occurred over the period.

453 Figure S3 (Supplemental Material) shows that the corresponding cumulative numbers of EQs

454 behave more linearly than as a sigmoid $(C$-factor $=1.1)$, and in any case more linearly than the

455 real case, confirming that the results obtained around the Nepal EQ in 2015 are not merely due 456 by chance.

\section{Conclusions}

In this paper we have analysed the Swarm satellite data around the time of 25 April 2015 Nepal EQ. Two different analyses suggest that this EQ involved a physical coupling between the

461 lithosphere and ionosphere in its preparation phase. The comparison of the Pc3 wave power of 462 the same two month period over three years (the EQ year together with the previous one and the 463 next one) shows some anomalous differences in the region around the EQ epicentre. A more 464 sophisticated technique based on the theory of critical systems, allows us to establish a clear 465 change in the behavior of the cumulative number of magnetic anomalies, which is analogous 466 with that found for the cumulative number of EQs occurred in the same region. This analogy 467 permits us to suggest that the potential LAI coupling would extend over a rather large interval of 468 time, i.e. around a month before and after the 2015 Nepal main-shock. This analysis is also 469 confirmed as not obtained by simple chance after a confutation analysis during an almost 470 aseismic 60-day period in the same region, but in the previous year. 
471 From the above analyses, it appears that the Nepal event probably affected the ionosphere before

472 the EQ occurrence.

473 We think that our paper represents an important step moving from the search of single 474 anomalies, which is still a difficult task, to the search of anomalous patterns, likely related to 475 EQs. The use of the cumulative number of anomalies is the solution we propose here, but we do 476 not exclude other strategies that can address better and more efficiently the same goal.

477 In addition, more case studies should be undertaken in some other tectonic settings. The reader is 478 reminded that the objective of the present analyses is not a retrospective prediction of an 479 impending EQ, rather an emphasizing of a potential LAI coupling during its preparation phase.

480 We would expect however, that the use of an accelerating power-law might be more suitable 481 than the sigmoid, though with some resulting frequent false alarms. A specific further study 482 should be done in order to assess whether information on the magnitude of the EQ could be 483 deduced from the number or the amplitude of the magnetic anomalies. In addition, only with the 484 simultaneous and combined analysis of many different physical parameters in the space, time 485 and frequency domains we can advance in this subject [De Santis et al., 2015]. A more detailed 486 analysis would require the investigation of some other large EQs; currently, this is beyond the 487 scope of this first Swarm data analysis, however this will be an important matter for future work.

\section{Acknowledgements}

490 This work has been undertaken in the frame of the ESA-funded SAFE (Swarm for Earthquake 491 Study) project under the STSE Swarm + Programme, and the INGV-funded project LAIC-U 492 (Lithosphere-Atmosphere-Ionosphere Coupling Understanding), although what we present in the 493 paper is authors' own responsibility only and not by the funding agencies. The data used are 
494 listed in the footnotes of the article where they are cited, so we thank USGS and ESA, for 495 providing seismic and satellite magnetic data, respectively, and NOAA (USA) and World Data 496 Center for Geomagnetism in Kyoto (Japan), for providing the magnetic indices. We thank 497 Giovanni Occhipinti and an unknown referee for providing useful comments and suggestions 498 that improved the paper significantly. Constantinos Papadimitriou and Dedalo Marchetti are 499 thanked for some calculations and figures they made for this paper.

\section{References}

502

503

Akaike, H. (1973), Information theory and an extension of the maximum likelihood principle, in Petrov, B.N., Csáki, F., 2nd International Symposium on Information Theory, Tsahkadsor, Armenia, USSR, September 2-8, 1971, Budapest: Akadémiai Kiadó, p. 267281.

Avouac, J.-P., Meng, L., Wei, S., Wang, T., and J.-P. Ampuero (2015), Lower edge of locked Main Himalayan Thrust unzipped by the 2015 Gorkha earthquake, Nature Geoscience, 8, 9, 708-711, http://dx.doi.org/10.1038/ngeo2518

Balasis, G., and M. Mandea (2007), Can electromagnetic disturbances related to the recent great earthquakes be detected by satellite magnetometers?, Tectonophysics, 431, 173-195, doi:10.1016/ j.tecto.2006.05.038.

Balasis, G., Daglis, I. A., Zesta, E., Papadimitriou, C., Georgiou, M., Haagmans, R. and Tsinganos, K. (2012), ULF wave activity during the 2003 Halloween superstorm: multipoint observations from CHAMP, Cluster and Geotail missions, Ann. Geophys., 30, 1751-1768, doi:10.5194/angeo-30-1751-2012. 
Balasis, G., Daglis, I.A., Georgiou, M., Papadimitriou, C., Haagmans, R. (2013), Magnetospheric ULF wave studies in the frame of Swarm mission: a time-frequency analysis tool for automated detection of pulsations in magnetic and electric field observations, Earth Planets and Space, 65, 1385-1398.

Balasis, G., C. Papadimitriou, I. A. Daglis, and V. Pilipenko (2015), ULF wave power features in the topside ionosphere revealed by Swarm observations, Geophys. Res. Lett., 42, 69226930, doi:10.1002/2015GL065424.

Bowman, D.D., G. Ouillon, C.G. Sammis, A. Sornette, and D. Sornette (1998), An observational test of the critical earthquake concept. J. Geophys. Res. 103, 24,359-24,372.

Cavanaugh, J.E. (1997), Unifying the derivations of the Akaike and corrected Akaike information criteria, Statistics \& Probability Letters, 31, 201-208.

Chen, S., M. Liu, L. Xing, W. Xu, W. Wang,Y. Zhu, and H. Li (2016), Gravity increase before the 2015Mw 7.8 Nepal earthquake, Geophys. Res. Lett., 43, 111-117, doi:10.1002/2015GL066595.

Contoyiannis, Y., S. M. Potirakis, K. Eftaxias, M. Hayakawa, and A. Schekotov (2016), Intermittent criticality revealed in ULF magnetic fields prior to the 11 March 2011 Tohoku earthquake (Mw=9), Physica A, 452, 19-28, doi:10.1016/j.physa.2016.01.065.

Currie, J. L., and C. L. Waters (2014), On the use of geomagnetic indices and ULF waves for earthquake precursor signatures, J. Geophys. Res. Space Physics, 119, 992-1003, doi:10.1002/2013JA019530.

De Santis, A. (2014), Geosystemics, entropy and criticality of earthquakes: a vision of our planet and a key of access, in "Nonlinear phenomena in Complex Systems: from Nano to Macro 

Scale” ed. E. Stanley and D. Matrasulov, NATO Science for Peace and Security Series C: Environmental Security, 3-20.

De Santis, A., G. De Franceschi, L. Spogli, L. Perrone, L. Alfonsi, E. Qamili, G. Cianchini, R. Di Giovambattista, S. Salvi, E. Filippi, F.J. Pavón-Carrasco, S. Monna, A. Piscini, R. Battiston, V. Vitale, P.G. Picozza, L. Conti, M. Parrot, J.-L. Pinçon, G. Balasis, M. Tavani, A. Argan, G. Piano, M.L. Rainone, W. Liu, and D. Tao (2015), Geospace perturbations induced by the Earth: The state of the art and future trends, Physics and Chemistry of the Earth, Parts A/B/C, Volumes 85-86, 2015, Pages 17-33, http://dx.doi.org/10.1016/j.pce.2015.05.004.

Dobrovolsky, I.P., S.I. Zubkov, and V.I. Miachkin (1979), Estimation of the size of earthquake preparation zones, Pure and Applied Geophysics, 117, 5, pp. 1025-1044, http://dx.doi.org/10.1007/BF00876083.

Fraser-Smith, A. C., A. Bernardi, P. R. McGill, M. E. Ladd, R. A. Helliwell, and O. G. Villard (1990), Low-frequency magnetic field measurements near the epicenter of the Ms 7.1 Loma Prieta earthquake, Geophys. Res. Lett., 17, 1465-1468, doi:10.1029/GL017i009p01465.

Feldl, N., and R. Bilham (2006), Great Himalayan earthquakes and the Tibetan plateau, Nature, $444,165-170$.

Freund, F.T. (2011), Pre-earthquake signals: underlying physical processes. J. Asian Earth Sci. $41,383-400$.

Friis-Christensen, E., H. Lühr, and G. Hulot (2006), Swarm: A constellation to study the Earth's magnetic field, Earth, Planets and Space, vol. 58, (4), p. 351-358. 
Galetzka, J., D. Melgar, J.F. Genrich, J. Geng, S. Owen, E.O. Lindsey, X. Xu, Y. Bock, J.-P. Avouac, L.B. Adhikari, B.N. Upreti, B. Pratt-Sitaula, T.N. Bhattarai, B.P. Sitaula, A. Moore, K.W. Hudnut, W. Szeliga, J. Normandeau, M. Fend, M. Flouzat, L. Bollinger, P. Shrestha, B. Koirala, U. Gautam, M. Bhatterai, R. Gupta, T. Kandel, C. Timsina, S.N. Sapkota, S. Rajaure, and N. Maharjan (2015), Slip pulse and resonance of the Kathmandu basin during the 2015 Gorkha earthquake, Nepal, Science, 349, 1091-1095, doi: 10.1126/science.aac6383

Galguly, N.D. (2016), Atmospheric changes observed during April 2015 Nepal earthquake, Journal of Atmospheric and Solar-Terrestrial Physics, 140, 16-22.

Jacobs, J. A., Kato, Y., Matsushita, S., and Troitskaya, V. A.: Classification of geomagnetic micropulsations, J. Geophys. Res., 69, 180-181, 1964.

Hayakawa, M., R. Kawate, O. A. Molchanov, and K. Yumoto (1996), Results of ultra-lowfrequency magnetic field measurements during the Guam earthquake of 8 August 1993, Geophys. Res. Lett., 23(3), 241-244, doi:10.1029/95GL02863.

Hayakawa, M., A. Schekotov, S. Potirakis, and K. Eftaxias (2015), Criticality features in ULF magnetic fields prior to the 2011 Tohoku earthquake, Proc. Japan Acad. B, 91, 25-30, doi:10.2183/pjab.91.25.

Heilig, B., H. Lühr, and M. Rother (2007), Comprehensive study of ULF upstream waves observed in the topside ionosphere by CHAMP and on the ground, Ann. Geophys., 25, $737-754$. 
Heilig, B., and P. R. Sutcliffe (2016), Coherence and phase structure of compressional ULF waves at low-Earth orbit observed by the Swarm satellites, Geophys. Res. Lett., 43, 945951, doi:10.1002/2015GL067199.

Kon, S., Nishihashi, M., Hattori, K. (2011), Ionospheric anomalies possibly associated with M6.0 earthquakes in the Japan area during 1998-2010: case studies and statistical study. J. Asian Earth Sci. 41, 410-420.

Li, M., and M. Parrot (2013), Statistical analysis of an ionospheric parameter as a base for earthquake prediction, J. Geophys. Res. Space Physics, 118, 3731-3739, doi:10.1002/jgra.50313.

Liu, J.Y., Chen, Y.I., Chuo, Y.J., Chen, C.S. (2006), A statistical investigation of preearthquake ionospheric anomaly. J. Geophys. Res. 111, A05304. http://dx.doi.org/10.1029/2005JA011333.

Maus, S., H. Lühr, M. Rother, K. Hemant, G. Balasis, P. Ritter, and C. Stolle (2007), Fifthgeneration lithospheric magnetic field model from CHAMP satellite measurements, Geochem. Geophys. Geosyst., 8, Q05013, doi:10.1029/2006GC001521.

Menk, F. (2011), Magnetospheric ULF Waves: A Review, in: “The Dynamic Magnetosphere”, edited by: Liu, W. and Fujimoto, M., IAGA Special Sopron Book Series, Springer, 3, 223.

Němec, F., O. Santolik, M. Parrot, and J.J. Berthelier (2008), Spacecraft observations of electromagnetic perturbations connected with seismic activity, Geophys. Res. Lett., 35, L05109, doi:10.1029/2007GL032517. 
601 Occhipinti, G., L. Rolland, P. Lognonne', and S. Watada (2013), From Sumatra 2004 to Tohoku-

602 Oki 2011: The systematic GPS detection of the ionospheric signature induced by 603 tsunamigenic earthquakes, J. Geophys. Res. Space Physics, 118, doi:10.1002/jgra.50322.

604 Parameswaran, R.M., T. Natarajan, K. Rajendran, C.P. Rajendran, R. Mallick, M. Wood, and

605

606

607

608

609

610

611

612

613

614

615

616

617

618

619

620

621
H.C. Lekhak (2015), Seismotectonics of the April-May 2015 Nepal earthquakes: An assessment based on the aftershock patterns, surface effects and deformational characteristics, Journal of Asian Earth Sciences, Volume 111, 161-174. http://dx.doi.org/10.1016/j.jseaes.2015.07.030.

Parrot, M. (1995), Use of satellites to detect seismo-electromagnetic effects. Adv. Space Res., 15, 27-35.

Piša, D., F. Nĕmec, O. Santolik, M. Parrot, and M. Rycroft (2013), Additional attenuation of natural VLF electromagnetic waves observed by the DEMETER spacecraft resulting from preseismic activity, J. Geophys. Res. Space Physics, 118, doi:10.1002/jgra.50469.

Plastino, W., Bella, F., Catalano, P.G., Di Giovambattista, R., 2002. Radon groundwater anomalies related to the Umbria-Marche, September 26, 1997, Earthquake. Geofis. Int. $41(4), 369-375$.

Potirakis, S., K. Eftaxias, A. Schekotov, H. Yamaguchi, and M. Hayakawa (2016), Criticality features in ultra-low frequency magnetic fields prior to the 2013 M6.3 Kobe earthquake, Annals of Geophysics, 59 (3), S0317, doi:10.4401/ag-6863.

Pulinets, S.A., and K.A. Boyarchuk (2004), Ionospheric Precursors of Earthquakes. Springer Verlag. 
622 Pulinets, S.A., and D. Ouzounov (2011), Lithosphere-Atmosphere-Ionosphere Coupling (LAIC)

623 model. An unified concept for earthquake precursors validation. J. Asian Earth Sci. 41, $624 \quad 371-382$.

625 Saito, T. (1969), Geomagnetic Pulsations, Space Sc. Rev., 10, 3, 319-412.

626 Scholz, C.H. (2002). The Mechanics of Earthquake and Faulting, vol. xxiv. Cambridge Univ.

627

628

630

631

632

633

634

635

636

637

638

639

640

641

642 Press, Cambridge/New York (471 pp.).

Sorokin, V.M., Chmyrev, V.M., Yaschenko, A.K. (2001), Electrodynamic model of the lower atmosphere and the ionosphere coupling. J. Atmos. Sol. Terr. Phys. 63, 1681-1691.

Stolle, C., H. Lühr, M. Rother, and G. Balasis (2006), Magnetic signatures of equatorial spread F as observed by the CHAMP satellite, J. Geophys. Res., 111, A02304, doi:10.1029/2005JA011184.

Torrence C. and G. P. Compo, (1998), A Practical Guide to Wavelet Analysis. Bull. Amer. Meteor. Soc., 79, 61-78.

Vizzini F., Brai M. (2012), In-soil radon anomalies as precursors of earthquakes: a case study in the SE slope of Mt. Etna in a period of quite stable weather conditions. J. Environ. Radioact., 113, 131-141.

Walker, S. N., V. Kadirkamanathan, and O. A. Pokhotelov (2013), Changes in the ultra-low frequency wave field during the precursor phase to the Sichuan earthquake, DEMETER observations, Ann. Geophys., 31, 1597-1603, doi:10.5194/angeo-31-1597-2013.

Williams, A. B., Taylor, F. J., (1988), Electronic Filter Design Handbook, McGraw-Hill Inc., US. 
643 Wu Y, Jiang Z, Liang H, Chang L, Chen C, Zhu S, Zhao J, Du J (2016), Coseismic deformations 644 of the 2015 MW 7.8 Gorkha earthquake and interseismic strain accumulation in the 645 Himalayan tectonic belt and Tibetan plateau, Tectonophysics, 670, 144-154.

646 Zao B. (2016), April 2015 Nepal earthquake: observations and reflections, Nat. Hazards, 80, $647 \quad$ Issue 2, 1405-1410.

648 